\title{
Prof. em. Dr. med. Hans-Diedrich Cremer
}

Am 18. April 1995 verstarb im Alter von 85 Jahren Dr. med. HansDiedrich Cremer, emeritierter Professor für Menschliche Ernährungslehre und langjähriger Direktor des Instituts für Ernährungswissenschaft der Justus-LiebigUniversität Giessen.

H.-D. Cremer studierte in Bonn, Kiel, Innsbruck und Köln Medizin und war nach Promotion und Habilitation für Physiologische Chemie von 1945-1956 an den Universitäten Heidelberg und Mainz als Dozent und apl. Professor tätig. Dort, insbesondere im Arbeitskreis um $\mathrm{K}$. Lang, entwickelte sich sein besonderes Interesse für die ,Ernährungswissenschaft", das im November $1956 \mathrm{zu}$ seiner Berufung auf den Lehrstuhl für ,,Menschliche Ernährungslehre" an der damaligen Justus-Liebig-Hochschule in Giessen führte. Er war der erste Inhaber eines derartigen Lehrstuhls in Deutschland.

Aus kleinsten Anfängen heraus baute er das erste „Institut für Ernährungswissenschaft" an einer deutschen Universität auf, zunächst im Rahmen der Humanmedizinischen Fakultät, ab 1970 in einem eigenen Fachbereich „Ernährungswissenschaften" der Justus-LiebigUniversität Giessen. Die Forschungsaktivitäten dieses Instituts erstreckten sich von Beginn an auf so verschiedene Bereiche wie Ernäh- rungsfaktoren bei Zahn- und Knochenbildung, Ätiologie der Zahnkaries, industrielle Behandlung von Lebensmitteln als Problem des Ernährungsphysiologen, Bedeutung der Darmflora für die Versorgung mit Vitaminen sowie Toxizität von Fremdstoffen in der Nahrung.

1961 folgte H.-D. Cremer für zwei Jahre dem Ruf auf die Stelle eines Leiters der Abteilung für Angewandte Ernährungswissenschaft der Ernährungs- und Landwirtschaftsorganisation der Vereinten Nationen (FAO) in Rom. Seit dieser Zeit waren Welternährungsprobleme, insbesondere Ernährungsprobleme in Entwicklungsländern, stets sein ganz besonderes Anliegen. Zahlreiche ausgedehnte Studienund Vortragsreisen führten ihn nach Afrika, Asien und Lateinamerika.

H.-D. Cremer war Autor bzw. Mitautor von über 250 wissenschaftlichen Publikationen und Büchern sowie Mitherausgeber, u.a. des „,Handbuchs der Ernährungslehre und Diätetik" sowie des ,Handbuchs der Landwirtschaft und Ernährung in den Entwicklungsländern". Als langjähriges Mitglied des Präsidiums und in zahlreichen wissenschaftlichen Ausschüssen leistete er wichtige Beiträge zur Arbeit der Deutschen Gesellschaft für Ernährung (DGE), die ihm 1980 die Ehrenmitgliedschaft verlieh. Er war gleichfalls Ehrenmitglied des American Institute of Nutrition sowie Mitglied zahlreicher weiterer nationaler und internationaler Institutionen und Fachgesellschaften.

Das besondere Verdienst von H.-D. Cremer war zweifellos sein unermüdlicher und tatkräftiger Einsatz für die wissenschaftliche und hochschulpolitische Anerkennung des Fachgebiets ,Ernährungswissenschaft". Er war maßgeblich an der Aufnahme des Studiums der ,Haushalts- und Ernährungswissenschaften" an der Universität Giessen (1963/64) beteiligt. Damit wurde im deutschsprachigen Raum erstmals die Möglichkeit geschaffen, im Rahmen eines Universitätsstudiums eine vollakademische Ausbildung in Ernährungswissenschaften zu vermitteln.

H.-D. Cremer, Träger des Bundesverdienstkreuzes, war ein Hochschullehrer, der es in hervorragender Weise verstanden hat, seine zahlreichen Mitarbeiter und Schüler zu fördern, ihnen Selbständigkeit $\mathrm{zu}$ geben und sie zu motivieren. Die Entwicklung der Ernährungswissenschaft als eigenständiges Fach an Universitäten im Deutschland der Nachkriegszeit wird mit seinem Namen verbunden bleiben.

Prof. Dr. Erich Menden Giessen 